plan $P$. Ce fait a pour effet d'introduire dans les formules des termes dépendant de la distance de $M$ au plan $P$. De plus dans les formules relatives à la détermination d'un point d'où l'on voit le milieu de l'éclipse à l'horizon, intervient aussi la dérivée $h^{\prime}$ de $h$ par rapport au temps, introduisant elle aussi d'autres termes.

Dans cette note je me propose de nontrer qu'on peut conduire le calcul de la réfraction horizontale de manière à faire disparaître tous ces termes.

2. Soit $D$ le point où la droite $S M^{\prime}$ intercepte le plan $P$ et désignons par $C$ le point où la droite $O D$ intercepte la surface terrestre. Il est évident que le point $D$ se comporte exactement comme le point $\boldsymbol{M}^{\prime}$. Abstraction faite de la réfraction terrestre, du point $D$ on verrait le phénomène exactement sous le même aspect, qu'on le voit réellement de $M$, modifié par la réfraction.

Désignons par $\varpi$ la parallaxe horizontale du soleil et posons:

$$
j=C D / O C=C D \quad x=O S C \text {. }
$$

Dans le calcul de $j$ on peut évidemment négliger l'aplatissement terrestre et faire $O C=\mathrm{I}$, comme on le fait aussi dans le calcul de $h$.

Menons encore la tangente $S B$ à la Terre. Les deux triangles $S O C$ et $S O B$ donnent

$$
\operatorname{tg} x=O C / O S \quad \sin \varpi=O B / O S .
$$

Mais on a $B O C==\varpi<9^{\prime \prime}$. Les deux points $C$ et $B$ sont donc très rapprochés. D'autre part l'aplatissement terrestre est bien petit. On peut donc profiter de ces circonstances pour admettre $O C=O B$. Il s'en suit que

$$
\operatorname{tg} x=\sin \varpi \quad \text { ou } \quad x=\varpi
$$

(2)

aux termes en $\sin ^{3} \varpi<10^{-13}$ près.

Désignons encore par $i$ l'angle $C S D$. On a alors

$$
\begin{aligned}
& O D=\mathrm{I}+j=O S \operatorname{tg}(x+i) \\
& O C=\mathrm{I}=O S \operatorname{tg} x
\end{aligned}
$$

et on en déduit $1+j=\operatorname{tg}(x+i) \operatorname{ctg} x$

ou en ayant égard à la relation (2)

$$
\mathrm{I}+j=\sin (\varpi+i) \operatorname{cosec} \varpi .
$$

D'autre part le triangle SOM donne $\mathbf{I}+h=O S \sin (x+i) \operatorname{cosec} z_{1}=\sin (\varpi+i) \operatorname{cosec} \varpi \cdot \operatorname{cosec} z_{1}(4)$ avec la même approximation. En rapprochant les relations ( $),(3),(4)$, on déduit facilement:

$$
\mathrm{I}+j=n \sin \left(z_{1}-\varrho\right)
$$

d'où l'on déduit par dérivation

$$
j^{\prime}=n \cos \left(z_{1}-\varrho\right) \cdot\left(z_{1}^{\prime}-\varrho^{\prime}\right)
$$

Observatoire de Bucarest, I9I4 Mai.
Mais à l'horizon on a $z_{1}-\varrho=90^{\circ}$, de sorte que les relations $(5)$ et $(6)$ donnent:

$$
\text { (A) }\left\{\begin{array}{l}
j=n^{-I} \\
j^{\prime}=0
\end{array}\right.
$$

$j$ est par conséquent égal à la constante de la réfractión.

Tous les termes, dont il fut question plus haut, disparaissent des formules correspondantes at point $D$. Ce point se trouve en effet dans le plan $P$, et de plus $h^{\prime}$ se trouve remplacé par $j^{\prime}$, qui d'après les relations (A) s'annule a l'horizon. - Pour déterminer le point $D$ on n'a donc qu'à se servir des formules relatives au point $M$ en y supprimant les termes dépendant de la distance de $M$ au plan $P$ et les termes contenant $h^{\prime}$ en facteur et en y remplaçant $h$ par $j$, constante de la réfraction.

3. Il nous reste à passer du point $D$, une fois déterminé au point $M T$ qui nous intéresse. A cette fin considérons

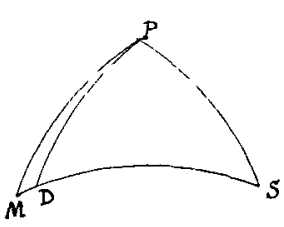

Fig. 2. (Fig. 2) sur la sphère céleste géocentrique le pôle $P$ et les points $S, D$, $M$ où elle est percée par les droites $O S, O M, O D$. Il est évident que ces trois points se trouvent sur le même arc de grand cercle, la réfraction ne changeant pas les azimuts. D'autre part on voit sur la figure I que l'on a arc $D M=D O M=\varrho_{0}-\varpi, \varrho_{0}$ étant la valeur de la réfraction à l'horizon.

Désignons par $H$ l'angle horaire du soleil sur le méridien d'origine - $H$ ne doit pas subir la correction de parallaxe - par $L$ la longitude de $D$ par rapport au méridien d'origine, comptée positivement vers l'Ouest, par $\varphi$ la latitude géocentrique de $D$, par $a, d$ les coordonnées équatoriales géocentriques du point $S$. On a alors (Fig. 2) $P S=90^{\circ}-d \quad P D=90^{\circ}-\varphi \quad S D=z \quad S P D==H-L$ de sorte que dans le triangle sphérique $P S D$ on a:

$$
\begin{aligned}
& \sin \varphi=\cos z \sin d+\sin z \cos d \cos P S D \\
& \sin z \sin P S D=\cos \varphi \sin (H-L) .
\end{aligned}
$$

L'arc $D M=\varrho_{0}-\varpi$ étant assez petit, on peut calculer les corrections $\mathcal{A} \varphi, \angle \mathcal{L}$, qu'on doit apporter aux coordonnées $\varphi, L$ de $D$ pour trouver celles de $M$, en différentiant les relations $(7)$ et en $\mathrm{y}$ faisant ensuite $z=90^{\circ}$. En remarquant que $t_{z}=\varrho_{0}-\varpi$, on trouve facilement

$$
\text { (B) }\left\{\begin{array}{l}
1 L=-\sin (H-L) \cos d \sec \varphi \cdot\left(\varrho_{0}-\varpi\right) \\
1 \varphi=-\sin d \sec \varphi \cdot\left(\varrho_{0}-\varpi\right)
\end{array}\right.
$$

relations à l'aide desquelles on passera des coordonnées $\varphi$, (6) $L$, du point $D$ à celles de $M T$.

\section{G. Demetrescu.}

\title{
Zur Vergleichung der Planeten- und Kometenephemeriden mit den Beobachtungen.
} Von S. Kasakou'.

Bei unserer Arbeit über den Kometen $1904 \mathrm{I}$ hatten wir ca. rooo Beobachtungen $z u$ reduzieren und mit der Ephemeride zu vergleichen. In Rechnungen dieser Art, die nicht schwierig, aber sehr zeitraubend sind, haben auch geringere Vereinfachungen große Wichtigkeit: So kann die Berechnung der parallaktischen Faktoren und die Berück- sichtigung'der Reduktion auf den scheinbaren Ort mit großer Zeitersparnis und genügender Genauigkeit graphisch durchgeführt werden.

Der parallaktische Faktor in AR. zum Übergang auf das Erdzentrum bei den üblichen Bezeichnungen lautet: $A=\varrho \pi \cos \varphi^{\prime} \sin (\theta-\alpha) \sec \delta$ 
wo man $\varrho=x$ anuehmen kann. Tragen wir auf der $x$-Achse eines rechtwinkeligen Achsensystems vom Anfangspunkte in einem willkürlich gewählten Maßstabe die Strecken $\pi \sec \delta$ $\mathrm{ab}$ und ziehen vom Anfangspunkte aus eir Strahlenbüschel so, daß die Tangente des Neigungswinkels einer Graden des Büschels gegen die $x$-Achse dem Sinus eines bestimmten Stundenwinkels gleich ist, so entspricht jedem Wertepaare $(\delta, \theta-\alpha)$ ein Punkt der Ebene, dessen Ordinate $\pi \sin (\theta-\alpha) \sec \delta$ ist. Um die Größe $A$ zu bekommen, ist diese Ordinate noch mit $\cos \varphi^{\prime} \mathrm{zu}$ multiplizieren, was auch einfach graphisch geschieht. Ziehen wir auf KoordinatenPauspapier eine Linie, die mit der Abszissen-Achse den Winkel $\varphi^{\prime}$ bildet, legen den Schnittpunkt dieser Linie mit der Abszissen-Achse auf den Fußpunkt der Ordinate und lassen dann die gezogene Linie mit der Ordinate zusammenfallen, so können wir auf der Abszissen-Achse des Koordinaten-Pauspapieres den Faktor $A$ unmittelbar ablesen. nation:

Im Ausdrucke des parallaktischen Faktors in Dekli-

$$
D=\varrho \pi \cos \delta \sin \varphi^{\prime}-\varrho \pi \sin \delta \cos \varphi^{\prime} \cos (\theta-\alpha)
$$

nehmen wir $\varrho=\mathrm{I}$ an und setzen:

also

$$
\begin{gathered}
x=\pi \cos \delta \quad y=\pi \cos (\theta-\alpha) \sin \delta \\
-D=-x \sin \varphi^{\prime}+y \cos \varphi^{\prime} .
\end{gathered}
$$

Man kann $x, y$ als Koordinaten eines Punktes der Ellipse mit den Halbachsen $\pi$ und $\pi \cdot \cos (\theta-\alpha) \mid$ betrachten; die Größen $\delta$ bei $\cos (\theta-\alpha)>0$ und $-\delta$ bei $\cos (\theta-\alpha)<0$ stellen den exzentrischen Winkel dieses Punktes dar. Der Faktor $D$ wird also dem absoluten Werte nach gleich, dem Vorzeichen nach entgegengesetzt der dem Punkte $\delta$ der Ellipse entsprechenden Ordinate in einem rechtwinkeligen Koordinatensysteme, dessen Achsen in bezug auf die Hauptachsen der Kurve um den Winkel $\varphi^{\prime}$ im entgegengesetzten Sinne des Uhrzeigers gedreht worden sind. Es ist aber leicht einzusehen, daß man nur ein Viertel jeder Ellipse zu zeichnen braucht, die Größe $\delta$ nur dem absoluten Werte nach nehmen, die Koordinatenachsen aber im Sinne des Uhrzeigers, oder entgegengesetzt drehen kann, je nachdem $\sin \delta \cos (\theta-\alpha)$ negativ oder positiv wird. Konstruieren wir die elliptischen Bogen für Stundenwinkel von $\circ^{\mathrm{h}}$ bis $6^{\mathrm{h}}$, etwa von $10^{\mathrm{m}} \mathrm{zu}$ Io ${ }^{\mathbf{m}}$ [wegen $\cos \left(6^{\mathrm{h}}+a\right)=-\cos \left(6^{\mathrm{h}}-a\right)$, so kann dieselbe Zeichnung auch für $6^{\text {h }}<\theta-\alpha<12^{\text {h }}$ dienen]; ziehen wir die Ordinaten für verschiedene Werte von $\delta$ von $\mathrm{I}^{\circ} \mathrm{zu} \mathrm{I}^{\circ}$, so wird jedem Paar $(\delta, \theta-\alpha)$ ein Punkt der Ebene entsprechen. Die Achsendrehung und die Ablesung der Ordinate kann wieder mittels des auf Koordinaten-Pauspapier gezeichneten Winkels $\varphi^{\prime}$ ausgeführt werden.

Mit den beschriebenen Nomogrammen kann man bei geringer Übung 50-75 Faktoren in der Stunde bekommen mit einer Genauigkeit von mindestens o".o5 bei einem Maßstabe I $0 \mathrm{~mm} / \mathrm{I}^{\prime \prime}$.

Bei der Berechnung der Differenzen der beobachteten und der berechneten Planeten- und Kometenkoordinaten vergleicht man gewöhnlich die beobachteten scheinbaren Örter mit den wahren, aus einer Ephemeride entnommenen fïr die Beobachtungszeiten die für Aberration korrigiert werden.

Beschäftigen wir uns zuerst mit visuellen MikrometerBeobachtungen. Die Ableitung der scheinbaren Koordinaten des Gestirnes verlangt die Reduktion der mittleren Vergleich- sternörter auf scheinbare. Nach dem Vorschlage von Kreutz (Untersuchungen über das Kometensystem …, I. Teil, S. 25 ) kann man die Arbeit dadurch erleichtern, daß man diese Reduktionen ephemeridenartig für die Ephemeridenörter berechnet und dann den Übergang auf die Sternörter mittels Differentialformeln beriucksichtigt. Es scheint uns aber, daß man der Berechnung dieser Reduktionen entsagen kann.

Nimmt man von den mittleren Ephemeridenkoordinaten $\alpha, \delta$ den Einfluß der Fixsternaberration ab, bildet also die neue Ephemeride, die

$$
\begin{aligned}
& \alpha^{\prime}=\alpha-h \sin (H+\alpha) \sec \delta \\
& \delta^{\prime}=\delta-h^{*} \cos (H+\alpha) \sin \delta-i \cos \delta
\end{aligned}
$$

enthält, korrigiert man ferner die Beobachtungszeiten für Aberration, so kann man die oben besprochenen Differenzen nach folgenden Formeln ableiten:

$$
\begin{aligned}
& \alpha_{0}+1 \alpha+1 M-\alpha^{\prime} \\
& \delta_{0}+1 \delta+\lambda N-\delta^{\prime}
\end{aligned}
$$

wo $\left(\alpha_{0}, \delta_{0}\right)$ die mittleren Koordinaten des Vergleichsternes, $(\boldsymbol{A} \alpha, \boldsymbol{\delta} \delta)$ die von dem Beobachter gegebenen Differenzen der scheinbaren Koordinaten des Gestirnes und des Vergleichsternes, $(1 M, 1 N)$ die Unterschiede der Reduktionen auf den scheinbaren Ort für die Koordinaten des Sternes und für diejenigen der Ephemeride sind. Wir beziehen $\left(\alpha_{0}, \delta_{0}\right)$ und $(\alpha, \delta)$ auf den Anfang des Beobachtungsjahres.

Sind die auf das mittlere Äquinoktium des Jahresanfanges bezogenen photographischen Orter eines Planeten oder Kometen gegeben, und bezeichnet man mit $\alpha_{0}$ und $M_{0}$ die mittlere Koordinate des Gestirnes und ihre Reduktion auf den scheinbaren Ort, dann wird $\alpha_{0}+M_{0}$ die scheinbare Koordinate des Himmelskörpers; der für die korrigierte Beobachtungszeit geltende wahre Ephemeridenort wird $\alpha^{\prime}+M$, wo $M$ wieder die Reduktion auf den scheinbaren Ort darstellt, daraus folgt die Differenz (Beobachtung-Rechnung):

$$
\left(\alpha_{0}-\alpha^{\prime}\right)+\Delta M
$$

Sind die Unterschiede $\left(\alpha_{0}-\alpha\right),\left(\delta_{0}-\delta\right)$ genügend klein, so können $\Lambda M, \Lambda N$ vernachlässigt werden und die mittleren beobachteten Koordinaten unmittelbar mit, unserer Ephemeride verglichen werden. Auf dieselbe Art behandeln wir die Meridiankreis-Beobachtungen.

Was aber die sog. säkulare Aberration anbetrifft, so kann man sie entweder an die Ephemeride anbringen (Ristenpart, Über Differentialreduktion …, A. N. I60.290), oder, was einfacher, nur in den Normalörtern beruicksichtigen.

Es bleibt uns noch übrig, die Ableitung von $\boldsymbol{M} M, d N$ zu erklären. Man kann diese Größen nach den Formeln von Kreutz (Op. cit.) berechnen, man kann auch die Hilfsgrößen von Ristenpart (Op. cit.) benutzen, aber bei einer großen Anzahl von Beobachtungen wird diese Arbeit unangenehm werden und es ist vorzuziehen auch hier eine graphische Methode anzuwenden, die uns rasch und sicher zum Ziele führt. Bezeichnen wir mit $M, N$ die Reduktionen auf den scheinbaren Ort der mittleren Ephemeridenkoordinaten $(\alpha, \delta)$ :

$$
\begin{aligned}
& M=f+g \sin (G+\alpha) \operatorname{tg} \delta+h \sin (H+\alpha) \sec \delta \\
& N=g \cos (G+\alpha)+h \cos (H+\alpha) \sin \delta+i \cos \delta .
\end{aligned}
$$

Bei nicht zu großen Werten von $\left(\alpha_{0}-\alpha, \delta_{0}-\delta\right)$, kann man sich mit den Differentialformeln begnügen: 


$$
\begin{aligned}
& 1 M=a\left(\alpha_{0}-\alpha\right)+b\left(\delta_{0}-\delta\right) \\
& L N=a_{1}\left(\alpha_{0}-\alpha\right)+b_{1}\left(\delta_{0}-\delta\right)
\end{aligned}
$$

wobei

$a=a^{\prime \prime} \cdot \operatorname{arc} \mathrm{I}^{\prime \prime}=[g \cos (G+\alpha) \operatorname{tg} \delta+h \cos (H+\alpha) \sec \delta] \operatorname{arc} \mathrm{I}^{\prime \prime}$ $b=b^{\prime \prime} \cdot \operatorname{arc} \mathrm{I}^{\prime \prime}=[g \sin (G+\alpha)+h \sin (H+a) \sin \delta] \sec ^{2} \delta \operatorname{arc~} \mathrm{I}^{\prime \prime}$ $a_{1}=a_{1}{ }^{\prime \prime} \cdot \operatorname{arc} \mathrm{I}^{\prime \prime}=-[g \sin (G+\alpha)+h \sin (H+\alpha) \sin \delta] \operatorname{arc~\mathrm {I}^{\prime \prime }}$ $b_{1}=b_{1}^{\prime \prime} \cdot \operatorname{arc} \mathrm{I}^{\prime \prime}=[h \cos (H+\alpha) \cos \delta-i \sin \delta] \operatorname{arc} \mathrm{I}^{\prime \prime}$.

Bei einer definitiven Bahnbestimmung, die wir besonders ins Auge fassen, sind die Ephemeridenkorrektionen gewöhnlich sehr klein und man kann im Falle von Mikrometer-Beobachtungen in die obigen Differentialformeln, statt $\left(\alpha_{0}-\alpha, \delta_{0}-\delta\right)$, die Größen $\mathcal{A} \alpha, \grave{A} \delta$ einführen. Bezeichnet man, der Kürze wegen, $d \alpha$ und $\not \delta$ mit $x$ und $y$, so wird

$\begin{aligned}-\mathrm{d} M & =a x+b y \\ \text { oder } & -\mathrm{d} M=\left[(a x+b y) / l^{\prime}\left(a^{2}+b^{2}\right)\right] V\left(a^{2}+b^{2}\right) .\end{aligned}$

Setzt $\operatorname{man} \quad a^{\prime \prime}=\varrho \cos \theta \quad b^{\prime \prime}=\varrho \sin \theta$

so bestimmen für $\varrho>0$ diese Gleichungen den Winkel $\theta$ eindeutig. Wir wollen vom Koordinatenanfangspunkte eines rechtwinkeligen Achsensystems aus einen Strahl, der unter dem Winkel $\theta$ gegen die $x$-Achse geneigt ist, ziehen und werden diesen Winkel von der positiven Richtung der $x$-Achse an von $0^{\circ}$ bis $360^{\circ}$ in einem bestimmten Sinne zählen. Der absolute Betrag von $(a x+b y) / l^{\prime}\left(a^{2}+b^{2}\right)$ ist dann die Länge der Strecke, die auf der Richtung des Strahles, oder auf der entgegengesetzten Richtung, zwischen dem Koordinatenanfangspunkt und dem Fußpunkt des Perpendikels aus dem Punkte $(x, y)$ liegt. Fällt der Fußpunkt dieses Perpendikels in die Richtung des Strahles selbst, so wird die Länge der Strecke $p=(a x+b y) / \nu^{\prime}\left(a^{2}+b^{2}\right)$, im entgegengesetzten Falle hat $\operatorname{man} p=-(a x+b y) / v\left(a^{2}+b^{2}\right)$, also

$$
-. M= \pm p l^{\prime}\left(a^{2}+b^{2}\right) \text { oder }-. M= \pm p Q \operatorname{arc} \mathrm{I}^{\prime \prime} .
$$

Bezeichnen wir mit $\lambda$ die Anzahl der Bogensekunden für die Längeneinheit des gewählten Maßstabes, so finden wir . $1 M$ in Bogensekunden durch $-J M= \pm p \varrho \lambda \operatorname{arc} \mathrm{I}^{\prime \prime}$, oder in tausendsten Teilen der Zeitsekunde durch

$$
-A M= \pm(\text { I } 000 \lambda \text { arc I"/ } 5) \varrho p \text {. }
$$

Setzt man $\lambda=60^{\prime \prime}$, führt statt $\theta$ die Größe $\theta_{0}=\theta+180^{\circ}$

Moskau, I 9 I 4 Juni 28. ein und bezeichnet $\varrho \cdot[8.2876-10]$ mit $R$, so bekommt man

$$
I M= \pm p R \text {. }
$$

Die Größen $\theta_{0}, R$ hat man ephemeridenartig mit dreistelligen Logarithmen zu berechnen.

Um die Bestimmung der Strecke $p$ wirklich durchzuführen, zeichnen wir auf Koordinatenpapier rechtwinkelige Koordinatenachsen, wobei wir die positive $x$-Achse nach links, die positive $y$-Achse nach oben gerichtet annehmen. Um den Koordinatenanfangspunkt als Mittelpunkt beschreiben wir einen Kreis und teilen ihn mit einem. Transporteur in Grade von $0^{\circ}$ bis $360^{\circ}$ vom positiven Ende der $x$-Achse beginnend im entgegengesetzten Sinne des Uhrzeigers. Aus Koordinaten-Pauspapier schneiden wir einen zweiten Kreis von demselben Radius aus und lassen ibn sich um den Anfangspunkt des obengenannten Koordinatensystems als Drehungsmittelpunkt drehen, so zwar, daß die Umdrehungsachse auch durch den Mittelpunkt des Pauspapierkreises geht. Nehmen wir diesen Mittelpunkt als Anfangspunkt eines zweiten Koordinatensystems von derselben Anordnung, wie das erste, an. In diesem zweiten beweglichen System markieren wir nach den gegebenen Werten $\boldsymbol{A} \alpha$ und ${ }_{2} \boldsymbol{\delta}$ in einem gewissen Maßstabe einen Punkt und drehen die Achsen so, daß das positive Ende der beweglichen $x$-Achse die Ablesung $\theta_{0}$ im geteilten Kreise anzeige, dann gibt in bezug auf das erste Achsensystem die Abszisse des markierten Punktes den Wert von $p$ und das Vorzeichen von $1 M$. Die Multiplikation von $p$ mit $R$ geschieht ganz leicht mit einem Rechenschieber, oder mit Zimmermanns. Rechentafel.

Die Berechnung von $d N$ wird in derselben Weise ausgeführt. Man setzt

$$
\begin{aligned}
a^{\prime \prime}{ }_{1}=\varrho_{1} \cos \theta_{1} & b^{\prime \prime}{ }_{1}=\varrho_{1} \sin \theta_{1} \\
\theta_{0} & =\theta_{1}+\mathrm{I} 80^{\circ} \quad R=\varrho_{1}[8.46 .37-\mathrm{Io}]
\end{aligned}
$$

und erhält . $I N$ in $0^{\prime \prime \prime o ~ I ~ d u r c h ~}$

$$
\Delta N= \pm p R \text {. }
$$

Bei gegebenen Werten $\theta_{0}, R$ kann man nach dieser Methode ca. roo Größen $\boldsymbol{I} M$ oder $\boldsymbol{I N}$ per Stunde bekommen mit einer Genauigkeit von mindestens 0.005 in AR. und 0.05 in $\delta$, beim Maßstabe $\lambda=60^{\prime \prime}$ auf $1 \mathrm{~mm}$.

\section{S. Kasakou'.}

\section{Besondere Fälle bei definitiven Bahnbestimmungen aus einer Erscheinung. Von S. Scharbe.}

Bei einer definitiven Bahnbestimmung aus einer Erscheinung kommen Fälle vor, in denen eine oder mehrere Unkekannte nicht sicher bestimmt werden können, weil ihr Koeffizient in den Eliminationsgleichungen zu klein heraus. kommt. Man kann die Frage stellen, wann solche Falle eintreten. Hier wollen wir versuchen auf diese Frage eine vorläufige Antwort zu reben.

Dazu werden wir die Aufgabe möglichst vereinfachen. 1. Nehmen wir an, daß wir nur drei vollständige Normalörter zu den Zeiten $t_{0}, t_{1}$ und $t_{z}{ }^{1}$ ) haben und aus den sechs gegebenen Differenzen der sphärischen Koordinaten $\mathrm{d} \lambda$ und $\mathrm{d} \boldsymbol{\beta}$ sechs Korrektionen zu den angenommenen Konstanten suchen. 2. Als Unbekannte wählen wir die Kor- rektionen der drei Anfangskoordinaten $\mathrm{d} x_{0}, \mathrm{~d} y_{0}, \mathrm{~d} z_{0}$ und der Projektionen der Anfangsgeschwindigkeit $\mathrm{d} x_{0}^{\prime}, \mathrm{d} y_{0}^{\prime}, \mathrm{d} z_{0}^{\prime}$ zur Zeit $t_{0} \cdot{ }^{*}$ 3. Wir führen folgendes geozentrische Koordinatensystem ein (und ein ihm paralleles heliozentrisches): die $x$-Achse legen wir durch den geozentrischen Ort zur Zeit $t_{0}$ und die $x y$-Ebene tangential an die geozentrische Bahn des Himmelskörpers zu derselben Zeit $t_{0}$. Nennen wir $\lambda$ und $\boldsymbol{\beta}$ die sphärischen Koordinaten in unserem Koordinatensystem, so sind also zur Zeit $t_{0}: \lambda_{0}=0, \boldsymbol{\beta}_{0}=0$ und $\boldsymbol{\beta}_{0}^{\prime}=0$. 4. Wir führen die Bezeichnung $x=x\left(t-t_{0}\right)$ ein, nehmen an, daß $\tau$ eine kleine Größe erster Ordnung ist, und werden im folgenden nur die Glieder bis zur zweiten Ordnung behalten. Also ist:

\footnotetext{
1) Wir nehmen an, daß $t_{0} z$ wischen $t_{1}$ und $t_{2}$ liegt.
} 